\title{
ADA: Avatar Animado para o Moodle como Auxílio Docente
}

\author{
Isabella M. Freitas ${ }^{1}$, Sofia L. Costa Paiva ${ }^{2}$ \\ ${ }^{1}$ Instituto de Ciências Exatas - Universidade Federal de Minas Gerais (UFMG) \\ - Belo Horizonte, MG - Brasil \\ ${ }^{2}$ Departamento de Ciência da Computação \\ Universidade Federal de São João del-Rei (UFSJ) - São João del-Rei, MG - Brasil \\ isabellafreitas@ufmg.br, sofia@ufsj.edu.br
}

\begin{abstract}
Despite adding value to teaching, the Moodle Virtual Learning Environment (VLE) leads teachers to face difficulties in accomplish tasks and including resources. This paper presents a tool to aid the use of Moodle platform, called ADA. This tool aims at aid users in educational resources creation, especially Questionnaire and Workshop resources. Usability tests were done with volunteer teachers collaboration, evidencing that the tool achieved, satisfactorily, the expected results of efficiency. We expected that this tool arouse interest in taking advantage the Moodle resources since it brings benefits in terms of practicality and efficiency.
\end{abstract}

Resumo. Apesar de agregar valor no ensino, o Ambiente Virtual de Aprendizagem (AVA) Moodle leva os docentes a enfrentarem dificuldades para realização de tarefas e inclusão de recursos. Este trabalho apresenta uma ferramenta para auxiliar o uso da plataforma Moodle, chamada ADA. Esta ferramenta visa assessorar os usuários na criação de recursos educacionais, especialmente aos recursos de Questionário e Laboratório de Avaliação. Ensaios de usabilidade foram feitos com a participação de docentes voluntários, evidenciando que a ferramenta atingiu, satisfatoriamente, os resultados esperados de eficiência. Espera-se que esta ferramenta desperte o interesse em usufruir dos recursos do Moodle uma vez que traz benefícios em termos de praticidade e eficiência.

\section{Introdução}

Para Anísio Teixeira (1971), patrono da educação pública brasileira, a escola deveria ser um ambiente bonito, moderno e acolhedor [Nunes 2007]. Ao idealizar a modernidade escolar, contudo, Anísio pode não ter considerado que a escola, com o passar dos anos, seria também virtual. O recente Censo da Educação Superior ${ }^{1}$ disponibilizado pelo Instituto Nacional de Estudos e Pesquisas Educacionais Anísio Teixeira (INEP) mostra que, entre 2015 e 2017, mais de 300 mil novos alunos optaram pelo modelo de ensino a distância (EAD), somando-se aos mais de 1 milhão de estudantes deste modelo no Brasil.

Os Ambientes Virtuais de Aprendizagem (AVA) estão presentes em diversas instituições de ensino, como maneira de expandir o conteúdo apresentado em sala de aula ou substituí-la por completo. Como benefício, o AVA proporciona uma aprendizagem com custo reduzido, por não necessitar de estrutura física; um acesso amplo à aprendizagem e uma divisão clara de responsabilidades entre discentes e docentes (ou tutores).

\footnotetext{
${ }^{1}$ Disponível em: http://portal.inep.gov.br/web/guest/resumos-tecnicos 1
} 
Tomemos como exemplo da atuação do AVA, ainda, a pandemia declarada este ano pela Organização Mundial de Saúde (OMS). Segundo a Unescd? mais de 1 bilhão de alunos foram afetados pela paralisação total e/ou parcial das aulas presenciais, ação adotada por países para conter a propagação da COVID-19. No Brasil, para minimizar os efeitos sentidos pela falta de atividades escolares, diversas instituições acadêmicas tem recorrido as aulas on-line aplicadas através de AVAs, expandindo o ensino a distância.

Com uma estimativa de 79 milhões de usuários, a plataforma Moodle $]^{3}$ é um dos AVAs mais adotados pelas universidades brasileiras. Presente como ferramenta de apoio, introduz um espaço onde o professor é responsável pela adição de materiais de ensino e atividades para seus alunos em uma página específica da disciplina ofertada (criada pelo administrador do espaço Moodle de sua instituição).

Navegabilidade, conexão do sistema com o mundo real e complexidade de execução de tarefas, segundo [Nielsen and Molich 1990], são as principais características esperadas em Interfaces de Usuário (IU). Um levantamento realizado pelas autoras evidencia que diversos autores debatem as insatisfações de usuários com o Moodle de suas instituições [Neves et al. 2019, Sousa 2016, Harrati et al. 2016, Antonino and Freire 2018, Zago and Polino 2017], destacando a inabilidade da plataforma em compreender satisfatoriamente as características propostas por [Nielsen and Molich 1990]. Antes que a pandemia transformasse o ambiente virtual no principal recurso acadêmico, docentes já sentiam dificuldade em adaptar-se ao Moodle, deixando a desejar na beleza e acolhimento propostos por Anísio Teixeira.

Diante deste cenário, foi idealizado um avatar animado como um esquema de aperfeiçoamento proposto para a plataforma Moodle. A ferramenta de auxílio ao docente chamada ADA (Auxílio Docente Animado) deve fortalecer o papel do ambiente virtual dentro dos cursos de graduação e pós-graduação, propiciando um guia interativo para que docentes possam realizar diversas tarefas que antes possuíam dificuldade. A ferramenta foi avaliada em termos de eficácia com usuários reais utilizando-se técnicas bem estabelecidas de usabilidade, evidenciando seu benefício aos usuários do Moodle.

O artigo está organizado como segue: a Seção 2 apresenta o referencial teórico e trabalhos relacionados; a ferramenta ADA é apresentada na Seção 3, abordando sua arquitetura e funcionamento. A Seção 4 apresenta a avaliação da ferramenta. Por fim, a Seção 5 apresenta as considerações finais.

\section{Referencial Teórico}

Esta seção apresenta os fundamentos acerca de Recursos Educacionais voltadas ao Moodle, a fim de elucidar conceitos pertinentes para a compreensão do trabalho; ainda, discorre sobre os principais trabalhos relacionados.

\subsection{Recursos Educacionais}

Para elaboração de atividades acadêmicas, a plataforma Moodle apresenta recursos educacionais divididos em quatro categorias, sendo (i) Gerenciamento de Conteúdo, como Lição, Enquete, Glossário, Laboratório de Avaliação e Tarefa; (ii) Ferramentas de

\footnotetext{
${ }^{2}$ Disponível em: https://en. unesco.org/covid19/educationresponse

${ }^{3}$ Modular Object-Oriented Dynamic Learning Environment. Disponível em https://moodle.org
} 
Comunicação, como chats, Fóruns, Mensagens e Blogs; (iii) Ferramentas Informacionais, como Calendário, Arquivos e Pasta; e (iv) Ferramentas Administrativas, como Relatório de Notas, Frequência e Gerenciamento de Usuários.

Inúmeros dos 25 recursos da plataforma Moodle são desconhecidos aos usuários, segundo [Neves et al. 2019]. Com dados coletados através de uma pesquisa que envolveu 150 docentes da Universidade Federal de São João del-Rei (UFSJ), o trabalho ilustra a subutilização de atividades como Conteúdo Interativo H5P, SCORM/AICC e Ferramenta Externa e Laboratório de Avaliação. Além disso, mais de $30 \%$ dos respondentes afirmaram não conhecer recursos que aparentavam popularidade, como Questionário.

Os recursos de Questionário e Laboratório de Avaliação atingem resultados extraordinários no ensino de disciplinas pelo Moodle, mesmo sendo pouco empregados por docentes [Mozzaquatro and Medina 2008, Cunha-Araújo et al. 2012]. Ao auxiliar estudantes a entenderem o processo de avaliação de trabalhos escritos, uma turma de pósgraduação pôde motivar $95,24 \%$ dos participantes a produzir textos científicos após o uso do Laboratório de Avaliação em uma disciplina [Ugulino et al. 2009].

O Questionário, comumente utilizado para avaliação do conhecimento dos estudantes sobre um assunto, pode beneficiar a experiência acadêmica do estudante; em uma disciplina de Endodontia, por exemplo, $43,8 \%$ dos alunos mostraram-se satisfeitos com a nova variação de atividades desenvolvidas para a matéria, indicando o aprimoramento do ensino [Cunha-Araújo et al. 2012].

\subsection{Desenvolvimento de Plugins para o Moodle}

O desenvolvimento de plugins para o Moodle amplia as possibilidades de uso da plataforma, permitindo a implementação de novas utilidades [Santos et al. 2019]. Atualmente, 1620 plugins de 911 desenvolvedores diferentes estão disponíveis, com 381.000 downloads realizados 4 . Os plugins do Moodle estão organizados em tipos distintos, destacando-se o bloco 5 , responsável por exibir informações nos espaços destinados a eles.

Baseado em uma arquitetura cliente-servidor, o Moodle utiliza a linguagem PHP para o servidor e deixa livre a escolha da linguagem para o cliente. Entretanto, utiliza módulos da linguagem JavaScript (JS) para realizar o monitoramento de mudanças em arquivos de produção, levando os desenvolvedores a escolherem esta linguagem para módulos do cliente nos plugins. Os desenvolvedores devem, ainda, ter conhecimento prévio em Node.j ${ }^{6}$, pois o sistema realiza monitoramento e prevenção de falhas a partir de diversas de suas bibliotecas.

\subsection{Trabalhos Relacionados}

Chamado de Avatar Educação, o trabalho [Maciel et al. 2014] apresenta um avatar animado como um importante recurso de afetividade, no intuito de promover a interatividade com o usuário na plataforma Moodle. Os resultados indicam que o avatar motivou o uso dos discentes; entretanto, como não era responsável por guiar o usuário pela plataforma, não aprimorou a interação entre o usuário e o sistema.

\footnotetext{
${ }^{4}$ Disponível em: https://moodle.org/plugins/

${ }^{5}$ Utilizado neste trabalho. Para saber mais: https://docs.moodle.org/dev/Blocks.

${ }^{6}$ Disponível em: https: / / nodejs.org
} 
$\mathrm{Na}$ forma de um agente inteligente para AVA, [Paschoal et al. 2016] apresentam a ferramenta Ubibot, que oferece diversos aspectos de aprendizagem ubíqua aos estudantes. $\mathrm{O}$ trabalho dedica-se a explicar as etapas de desenvolvimento do agente, sem explorar a performance do agente em contato com os discentes. Ainda, foca no ensino da disciplina em que está alocado, sem auxílios voltados ao Moodle.

Em [Correia et al. 2015] temos um agente pedagógico animado tridimensional para suavizar a ausência física de um educador dentro de AVAs. Enquanto o personagem busca estreitar os laços entre docentes e discentes no ambiente virtual, os resultados descritos detalham sua concepção, mas não indicam sua implementação. Ainda, o foco do trabalho é ser uma presença virtual a fim de simular o papel docente.

\section{A Ferramenta ADA}

Esta seção apresenta a ferramenta ADA (Auxílio Docente Animado), planejada e desenvolvida como um plugin do Moodle. O desenvolvimento foi norteado por dois ideais para otimizar sua produção: a simplicidade e a conformidade com as regras da plataforma.

\subsection{Projeto e Desenvolvimento}

O projeto ADA iniciou com a prototipação da ferramenta. A fim de construir a identidade visual do avatar, possibilitando a análise estética e de interação com o sistema, um wireframe fora criado. O wireframe contribuiu, ainda, para a concepção de um menu de seleção drop-down que segue o fluxo do processo da criação dos recursos educacionais, permitindo a familiarização do docente com as opções disponíveis para configuração da página e uma rápida identificação do estado atual de configuração.

A partir do protótipo, foram escolhidas as tecnologias que viabilizaram o projeto, como tradução de texto para fala e geração de personagens animados; além disso, embora a ferramenta pudesse ser desenvolvida para todos os recursos educacionais presentes no Moodle, o projeto inicial optou por empregá-la no Questionário e no Laboratório de Avaliação, escolha norteada pelos dados presentes na Seção 2.1.

As tecnologias consideradas deveriam seguir alguns critérios como (i) licença gratuita; (ii) disponibilidade; (iii) tempo de processamento; (iv) necessidade de memória para execução; e (v) facilidade de uso. Duas bibliotecas JS corresponderam satisfatoriamente com os requisitos. A primeira, Responsive Voice 7 , é uma API desenvolvida com o intuito de transformar textos em fala, enquanto a segunda, Avataaars 8 , é uma biblioteca de geração de personagens ilustrados. Entre as opções de configuração, há elementos como cabelo e acessórios, o que permitiu a confecção da ADA seguindo preferências pessoais.

Para o desenvolvimento do plugin, a classe $h t_{m} l_{\text {_writer }} \mathrm{9}^{9}$ auxiliou a construção do menu e disposição do avatar na tela, já que o Moodle não permite arquivos do tipo . $\mathrm{html}$ em plugins. Desta classe, a implantação da interface do bloco usou as funções tag, start_tag e end_tag e img, link e script, todas semelhantes as etiquetas de mesmo nome presentes na linguagem HTML. Para o processo de animação e interação com o docente, as ações da API de fala foram incluídas ao projeto através de arquivos PHP, já que houve

\footnotetext{
${ }^{7}$ Disponível em: https://responsivevoice.org

${ }^{8}$ Disponível em: https://avataaars.com/.

${ }^{9}$ Permite escrever tags HTML básicas dentro de arquivos PHP.
} 
redução de funcionalidades JS empregadas pelo Moodle ${ }^{10}$. Embora as ações devam ser escritas em arquivos JS, esta mudança não interferiu na implantação do plugin.

Dispensou-se o uso de tecnologias para animação, a fim de não sobrecarregar a plataforma. Para criar a impressão da animação (afinal, o projeto indica Animado) a implementação troca as imagens do avatar, enquanto este vocaliza. Para realizar tal troca, uma função slide() é chamada múltiplas vezes em um curto intervalo de tempo, intercalando uma série de imagens inseridas ao plugin para este fim.

\subsection{Apresentação da Ferramenta}

As Figuras 1 e 2 apresentam a ferramenta inserida na página de configuração do recurso Questionário e Laboratório de Avaliação, respectivamente. As imagens evidenciam o sucesso da implantação do avatar no sistema, determinando ainda que não há interferência na navegação mesmo se encontrando em posição de destaque na IU.

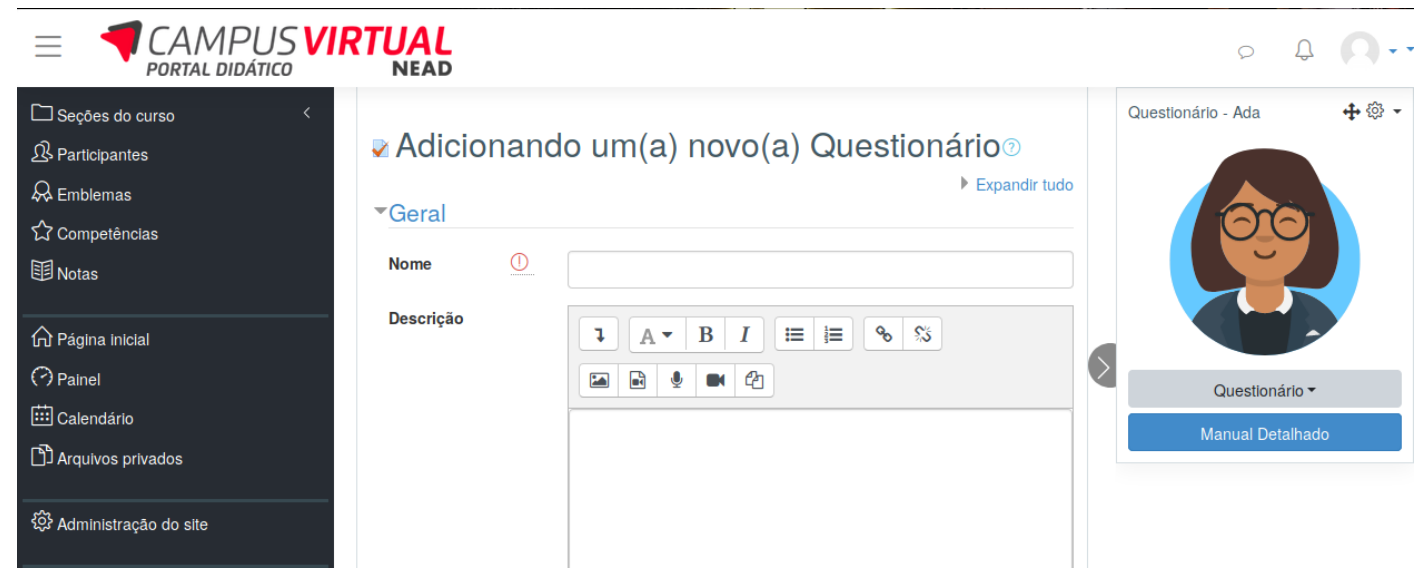

Figura 1. Presença da ADA na página de criação do Questionário.

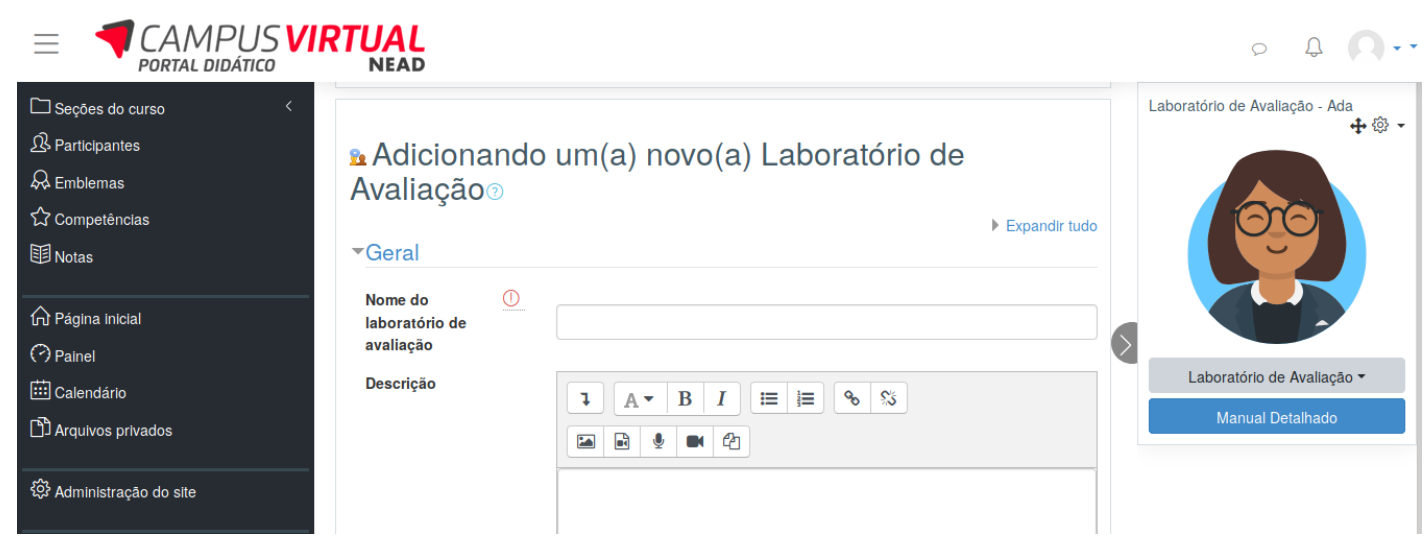

Figura 2. Presença da ADA na página de criação do Laboratório de Avaliação.

Para os docentes que preferem o auxílio não vocalizado, o plugin conta com um botão "Manual Detalhado", que leva a um texto sobre as configurações do recurso educacional. Os textos de ajuda utilizado nas falas da ADA e na construção do manual são

\footnotetext{
${ }^{10}$ A plataforma explica diversos motivos para essa decisão, como evitar lentidão do sistema e uso de APIs maliciosas.
} 
derivados dos documentos complementares do curso de capacitação "Moodle: possibilidades e criatividade na minha sala de aula" da Universidade de Brasília 11 .

Ao entrar na página de configuração do recurso, a ADA inicia a fala "Bem vindo, docente!". Escolhida uma opção do menu, digamos "O que é esse recurso?", a ADA dirá "Esta atividade permite ao professor criar e configurar testes de múltipla escolha, verdadeiro ou falso e outros tipos de perguntas. Cada tentativa é corrigida automaticamente e o professor pode optar por fornecer feedback elou mostrar as respostas corretas.' 12 .

\section{ADA: Avaliação da Ferramenta}

Esta seção apresenta os resultados obtidos na avaliação da eficácia da ferramenta com usuários reais. O planejamento do protocolo de avaliação foi realizado de modo a verificar quais aspectos são satisfatórios ou deficientes na ADA.

\subsection{Planejamento}

Conforme [Nielsen et al. 1994], os aspectos a serem considerados para testes de eficácia de projetos partem das metas a atingir; da seleção do universo amostral de usuários de teste e da definição das tarefas; dos dados a serem coletados, a maneira de análise após a coleta e o julgamento do sistema analisado. A Tabela 4.1 sumariza estes aspectos, ilustrando as etapas do ensaio de usabilidade aplicado à ADA.

\begin{tabular}{|c|l|}
\hline Etapa & \multicolumn{1}{c|}{ Descrição } \\
\hline \multirow{3}{*}{ Preparação } & $\begin{array}{l}\text { Verificação das condições a serem atendidas para realização do teste, sendo elas: } \\
\text { 1. Local; } \\
\text { 2. Sistema Computacional; } \\
\text { 3. Instruções e instrumentos para o questionário; }\end{array}$ \\
\hline \multirow{2}{*}{ Introdução } & $\begin{array}{c}\text { 1. Recepção do usuário e explicação breve dos propósitos do trabalho; } \\
\text { 2. Introdução aos procedimentos a serem adotados no teste; }\end{array}$ \\
& 3. Instruções e esclarecimento de dúvidas. \\
\hline \multirow{2}{*}{ Teste } & $\begin{array}{l}\text { 1. Observação imparcial dos eventos interativos usuário-sistema; } \\
\text { 2. Anotação de detalhes pertinentes ao contexto avaliatório; } \\
\text { 3. Diálogo limitado ao necessário com o avaliador. }\end{array}$ \\
\hline \multirow{2}{*}{ Análise dos Resultados } & $\begin{array}{l}\text { 1. Apresentação do questionário ao usuário de teste; } \\
\text { 2. Associação de todas as informações coletadas ao usuário; } \\
\text { 3. Elaboração do relatório final. }\end{array}$ \\
\hline
\end{tabular}

Tabela 1. Etapas do ensaio de usabilidade aplicado à ADA.

\subsection{Usabilidade e Experiência do Usuário}

Questionários são elaborados e largamente utilizados para coletar dados relevantes ao contexto de Usabilidade e Experiência do Usuário [Rauschenberger et al. 2013]. Neste trabalho foram selecionados o System Usability Scale (SUS) [Brooke et al. 1996] e o User Experience Questionnaire (UEQ) [Rauschenberger et al. 2013], que permitem analisar a percepção do usuário com a ferramenta avaliada. Para a aplicação dos questionários, houve a definição de participantes e das tarefas a serem realizadas, como descrito a seguir.

\footnotetext{
${ }^{11}$ Disponível em: https://moodle.ead.unb.br/mod/page/view.php?id=6694

${ }^{12}$ Para o caso, por exemplo, do recurso presente na Figura 1- Questionário.
} 
Participantes Foram convidados dez docentes alocados na Universidade Federal de São João del-Rei (UFSJ). Destes, cinco foram do Departamento de Ciência da Computação da Universidade, enquanto cinco foram de departamentos distintos que não poderiam estar ligados à cursos de tecnologia.

- Motivação: O diferente grau de conhecimento de tecnologias dá-se para enriquecer a discussão sobre as possibilidades de uso do avatar.

Tarefas Cada entrevistado foi orientado a criar quatro tarefas (duas sem usar o plugin, e duas usando o plugin ADA): (i) Laboratório de Avaliação; (ii) Laboratório de Avaliação usando ADA; (iii) Questionário; e (iv) Questionário usando ADA.

- Motivação: Quando a tarefa é realizada sem o avatar, posteriormente o docente pode focalizar sua interação nas dúvidas derivadas desta primeira atividade; o contato não é realizado de forma arbitrária.

\subsection{Resultados Obtidos}

Os participantes realizaram as tarefas em suas salas durante todo o tempo, para que o ambiente de trabalho fosse observado. A realização destas tarefas contribuiu para a coleta de diversos feedbacks dos docentes em relação ao uso da ADA. Alguns comentários sobre a ferramenta merecem destaque, como:

Participante 01: "Poderia apresentar uma janela com um texto descritivo com o mesmo conteúdo da fala do avatar."

Participantes 03 e 04: "Deveria haver uma maneira de pausar a voz do avatar, quando não se quiser mais ouvi-lo."

Participante 05: "Ela é muito simpática! Queria que estivesse em outras ferramentas do Moodle."

Participante 06: "Recurso deveras válido e necessário, que pode servir de incentivo para um uso mais pleno dos recursos."

Participante 07: "Parabéns pela iniciativa relevante e pertinente no auxílio do uso e da divulgação do portal."

O aprimoramento da interação com o Moodle culminou em elogios, como:

"É difícil que vocês da computação se interessem em ajudar os outros cursos.

Fico feliz que você (a avaliadora) esteja fazendo um trabalho voltado para a comunidade da Universidade, para os professores, para ajudar o nosso dia-a-dia."

Após sumarização dos resultados, a média foi de 61 pontos, pouco abaixo do esperado, já que as avaliações divergem no SUS, conforme a Figura 3 . Embora próxima da média de 68 pontos ${ }^{13}$, um melhor desempenho do sistema era esperado. Com total aversão ao sistema Moodle, apenas um docente desgostou de participar da avaliação e deu uma nota inferior a 50 pontos.

A análise das respostas fornecidas pela média SUS atingida pela ferramenta revelou que duas questões chamaram a atenção, sendo (i) Eu imagino que a maioria das pessoas iriam aprender a usar este recurso muito rapidamente e (ii) Eu precisava aprender um monte de coisas antes de começar a utilizar este recurso. Ao receberem Concordo Parcialmente/Totalmente de $90 \%$ dos participantes, mostram que os participantes encontraram dificuldade em utilizar a ferramenta dentro do contexto Moodle, mas não em utilizá-la como um recurso individual (de acordo com a primeira questão).

\footnotetext{
${ }^{13}$ Defendida por [Brooke et al. 1996] como a média de um bom sistema.
} 


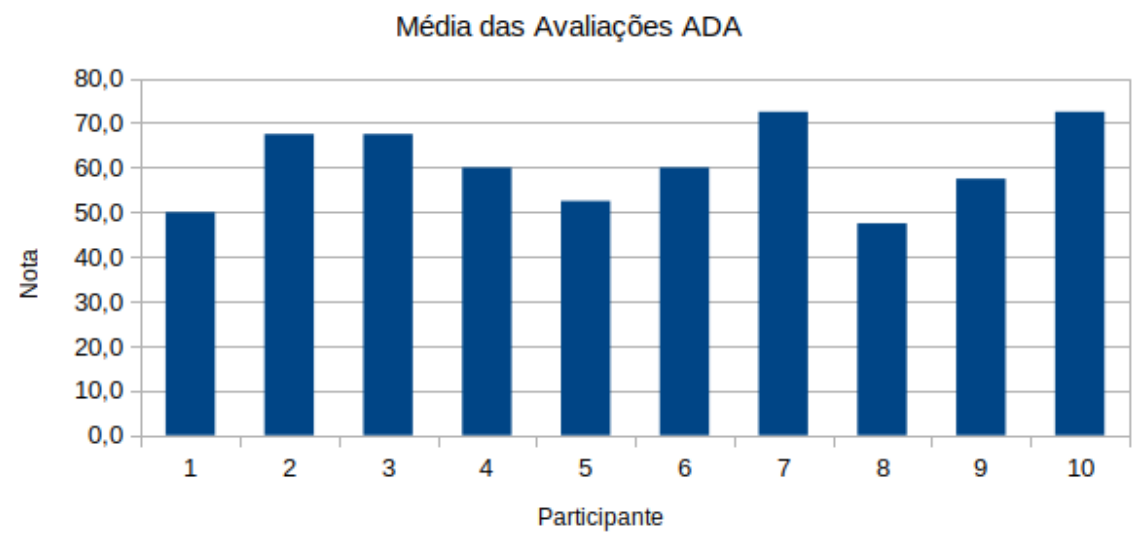

Figura 3. Avaliação de Usabilidade, analisada através do Questionário SUS.

Essa conclusão fora obtida, em parte, pelo feedback recebido durante a aplicação dos questionários. Um docente expressou que, embora a ferramenta fosse de fácil uso, chegar até ela era difícil e dependia de diversos conhecimentos acerca da plataforma Moodle. Isso afetou sua percepção sobre a ferramenta - e, por consequência, a maneira como respondeu as duas questões citadas acima. Esta opinião levanta a hipótese de auxílio em toda plataforma Moodle, o que pode aprimorar a utilidade e recepção da ferramenta ADA.

Para avaliar as características principais de um projeto, o questionário UEQ apresenta pares de adjetivos aos analisadores. Após a análise das respostas, estes adjetivos são transformados em índices de Atratividade, Transparência, Eficiência, Confiança, Estímulo e Inovação. A Figura 4 apresenta o resultado obtido, indicando bons índices de Eficiência e Estímulo, enquanto, infelizmente, o tópico de Inovação não se destacou.

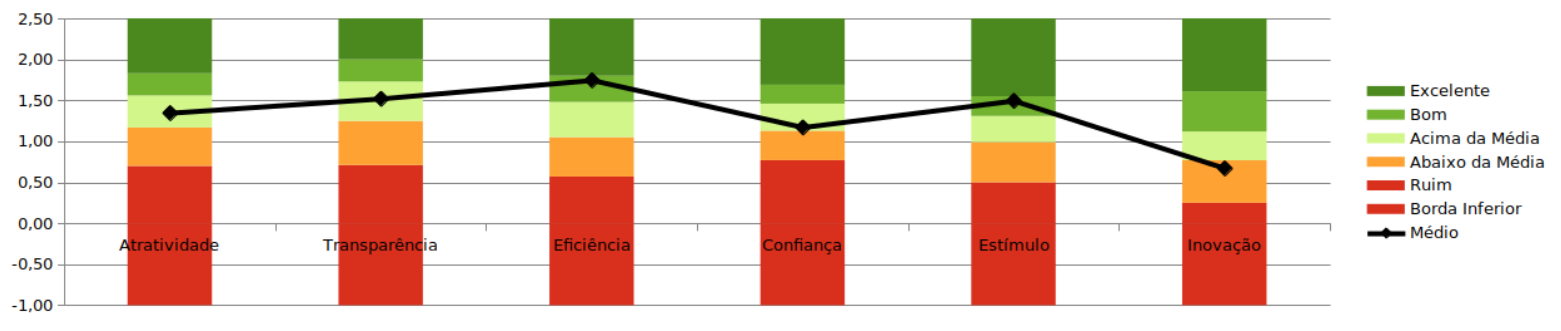

Figura 4. Escala de características encontradas na ADA, pelo Questionário UEQ.

Houve a impressão, por parte das autoras, que a maneira corriqueira em que avatares animados estão presentes em sistemas computacionai ${ }^{14}$ pode ter contribuído para a sensação de pouca originalidade. Ainda, a ferramenta está inserida em um ambiente conhecido pelos docentes, promovendo uma sensação de familiaridade com os elementos presentes na interface, mesmo que sejam novos.

A Confiança da ferramenta fora impactada pela falta de um botão para pausar o áudio. Alguns docentes interrompiam uma fala com outra, sobrepondo áudios maiores com áudios menores e fazendo a animação da fala continuar mesmo quando não há vocalização. Como a animação é realizada através de um callback da função de fala, o avatar se mexe mesmo quando não há necessidade.

\footnotetext{
${ }^{14}$ Quem não se lembra, por exemplo, do personagem de auxílio da Microsoft Word.
} 
Os participantes pontuaram, ainda, que as falas maiores podem dificultar a interação e agradabilidade da ADA, uma vez que estão acostumado a serem interrompidos durante o dia-a-dia, seja por outros colegas, seja por alunos, ou afazeres de natureza diversa. Docentes da área de tecnologia relataram, também, que gostariam de um áudio mais conciso - enquanto os docentes de outras áreas gostariam de áudios mais detalhados, que explicassem mais o contexto dos itens. Essa diferença pode ter origem do grau de familiaridade dos docentes com sistemas eletrônicos.

Para certos voluntários, o conceito claro do que deveria ser um avatar animado não fora atingido pela ferramenta, não se deixando impressionar. Isto pode afetar a percepção geral sobre a originalidade do projeto, o que impacta em sua Atratividade. Ainda, alguns voluntários esperavam mais funções, como identificar e corrigir erros, e não cientes das limitações tecnológicas de tais funções, escolheram características que impactaram na Eficiência da ferramenta. Embora existam pontos a serem trabalhados, a ferramenta fora amplamente aceita pelos docentes. Um dos voluntários acrescenta, ainda:

"Eu queria saber usar mais recursos, mas eu não tenho tempo de aprender a usar um por um, então só utilizo a tarefa e a atividade. A ferramenta vai me ajudar a explorar coisas novas."

\section{Considerações Finais}

Este trabalho apresentou o desenvolvimento de uma ferramenta com o intuito de aprimorar o vínculo entre docentes e a plataforma Moodle, um famoso ambiente AVA empregado em diversas universidades brasileiras. O projeto possibilitou o uso de recursos que antes eram atípicos para os docentes voluntários que participaram da fase de validação.

Trabalhos futuros poderão tratar alguns pontos citados pelos docentes, como a implantação de ADA em outros recursos do Moodle, recursos para controle da interação com o usuário e melhorias na animação usando outras formas de conduzi-la. Também foi evidenciada por mais de um participante a necessidade de um auxílio mais aprofundado.

A recepção positiva dos docentes à ADA estimula a concepção de novas funcionalidades a serem acrescentadas à ferramenta, como um texto alternativo a ser apresentado com a fala. Pode-se pensar, até mesmo, em estender sua presença para outras partes da plataforma. Além disso, pode-se pensar em explorar as características que receberam uma recepção abaixo do esperado na etapa de validação da ferramenta, fazendo com que este seja um trabalho em constante evolução. É seguro afirmar, entretanto, que a utilização da plataforma será estimulada pelo projeto, agregando maior valor a experiência acadêmica dos discentes de disciplinas em que o professor optar pelo uso amplo da ADA.

\section{Referências}

[Antonino and Freire 2018] Antonino, M. R. L. and Freire, E. S. S. (2018). Avaliação da usabilidade da interface do ambiente virtual moodle: Uma visão dos alunos do curso de licenciatura em computação. Anais do Computer on the Beach, pages 258-267.

[Brooke et al. 1996] Brooke, J. et al. (1996). Sus-a quick and dirty usability scale. Usability evaluation in industry, 189(194):4-7.

[Correia et al. 2015] Correia, C. L., Montané, F. A. T., and del Real Tamariz, A. (2015). Processo de criação e desenvolvimento de um agente pedagógico animado $3 \mathrm{~d}$ para ambientes virtuais de aprendizagem. InterSciencePlace, 1(29). 
IX Congresso Brasileiro de Informática na Educação (CBIE 2020)

Anais do XXXI Simpósio Brasileiro de Informática na Educação (SBIE 2020)

[Cunha-Araújo et al. 2012] Cunha-Araújo, I. M. Z., Salazar-Silva, J. R., D’Assunção, F. L. C., and de Melo, Â. B. P. (2012). Avaliação da percepção dos alunos da disciplina de endodontia sobre o uso do ambiente virtual de aprendizagem (moodle). uso do questionário de auto-avaliação colles. Revista da ABENO, 12(2):163-169.

[Harrati et al. 2016] Harrati, N., Bouchrika, I., Tari, A., and Ladjailia, A. (2016). Exploring user satisfaction for e-learning systems via usage-based metrics and system usability scale analysis. Computers in Human Behavior, 61:463-471.

[Maciel et al. 2014] Maciel, A. M., Rodrigues, R. L., and Carvalho, E. C. (2014). Desenvolvimento de um assistente virtual integrado ao moodle para suporte a aprendizagem online. In Brazilian Symposium on Computers in Education, volume 25, page 382.

[Mozzaquatro and Medina 2008] Mozzaquatro, P. M. and Medina, R. D. (2008). Avaliação do ambiente virtual de aprendizagem moodle sob diferentes visões: aspectos a considerar. RENOTE, 6(1).

[Neves et al. 2019] Neves, A. L., Paiva, S. C., Rocha, L., and Albergaria, E. (2019). Explorando recursos e atividades do moodle. In Anais do 25 Congr. Intern. ABED de Educação a Distância, 2019, pages 1-8. Associação Bras. de Educação a Distância.

[Nielsen et al. 1994] Nielsen, J., Mack, R. L., et al. (1994). Usability inspection methods, volume 1. Wiley New York.

[Nielsen and Molich 1990] Nielsen, J. and Molich, R. (1990). Heuristic evaluation of user interfaces. In Proc SIGCHI Conf Human Factors in Comp. Syst, pages 249-256. ACM.

[Nunes 2007] Nunes, C. (2007). Trajetória intelectual e identidade do educador: Anísio teixeira (1900-1971). Revista Brasileira de Estudos Pedagógicos, 81(197).

[Paschoal et al. 2016] Paschoal, L. N., Mozzaquatro, P., Krassmann, A. L., and Binelo, M. O. (2016). Ubibot: Agente inteligente consciente do contexto de aprendizagem do usuário integrado ao ambiente moodle. In XXI Congresso Internacional de Informática Educativa (TISE), pages 95-104.

[Rauschenberger et al. 2013] Rauschenberger, M., Schrepp, M., Cota, M. P., Olschner, S., and Thomaschewski, J. (2013). Efficient measurement of the user experience of interactive products. How to use the user experience questionnaire (ueq). example: Spanish language version. Int. Journal of Art. Intellig. and Interact. Multimedia. 2(1): 39-45.

[Santos et al. 2019] Santos, J. R., Pimentel, E., Dotta, S. C., and Botelho, W. (2019). Estudo comparativo de plugins moodle para análise e acompanhamento da aprendizagem. In Anais do XXX Simpósio Bras. de Informática na Educação (SBIE 2019). SBC.

[Sousa 2016] Sousa, M. I. d. (2016). Analisando a interface do moodle: problemas de usabilidade. Revista Multitexto, 4(01).

[Ugulino et al. 2009] Ugulino, W., Marques, A. d. M., Pimentel, M., and Siqueira, S. W. (2009). Avaliação colaborativa: um estudo com a ferramenta moodle workshop. $X X$ Simpósio Brasileiro de Informática na Educação, Florianópolis/SC.

[Zago and Polino 2017] Zago, G. d. S. and Polino, C. d. A. (2017). Interfaces, usabilidade e ambientes virtuais de aprendizagem: uma avaliação heurística do ava ufpel. Cadernos da Escola de Comunicação, 1(14). 